\title{
Aerochair
}

\section{Integrative design methodologies for lightweight carbon fiber furniture design}

\author{
Rebeca Duque Estrada ${ }^{1}$, Maria Wyller ${ }^{2}$, Hanaa Dahy ${ }^{3}$ \\ ${ }^{1,2}$ ITECH Master Program University of Stuttgart ${ }^{3}$ BioMat Department: Bio-Based \\ Materials and Materials Cycles in Architecture, Institute of Building Structures and \\ Structural Design, University of Stuttgart, 70174 Stuttgart, Germany \\ 1,2 \{duque.rebeca|mariawyller\}@gmail.com \\ 33.dahy@itke.uni-stuttgart.de \\ Carbon fiber composites embody lightweight and strength and is a \\ well-integrated material in various fields of engineering. In spite of its excellent \\ material properties, it is not frequently found in architecture and design \\ applications. In this project, the intention is to research how the material's most \\ prominent qualities can be applied to create a lightweight furniture design. The \\ furniture object was chosen as an example of a small architectural component \\ with a structural capacity of holding a human body weight between $60-90 \mathrm{Kg}$. In \\ particular, carbon fiber composites display an impressive tensile strength, and \\ with the aim of exploring this feature, a case-study of a full-scale, hanging carbon \\ chair was conducted. To develop a design, optimize it and realize it, an integrated \\ design and fabrication process was developed. It combined material research, \\ computational design, and a novel fabrication method for filament materials.
}

Keywords: carbon fiber composites, computational design, lightweight furniture, chair design, fiber winding

\section{INTRODUCTION}

Carbon fiber composites display excellent mechanical properties and have been valued within the fields of aerospace and civil engineering for decades. Even though it is not yet a widespread material in architecture and design, research done on coreless filament winding systems developed by the Institute of Computational Design (ICD) and the Institute of Building Structures and Structural Design (ITKE) at the University of Stuttgart shows how the qualities of com- posite filament materials can create material effective lightweight designs on an architectural scale (Prado et al. 2014).

In spite of all of its qualities, it is important to note that the package of resilience, lightweight and strength that it presents come at a cost. Carbon fiber composites are expensive compared to other material options, they are not biodegradable and are difficult to recycle. Humans working with it must wear protective gear such as masks and protective suits 
to avoid contact with the resin as well as the fiber strands. Even though carbon fiber is currently not considered an environmentally friendly material, the production of it consumes less energy compared to for example aluminum or steel.

In this project, the qualities of carbon fiber composites inspired the design of a lightweight piece of furniture, while its limitations motivated an effective use of the material and became the main design driver. By exploiting the materials dominant qualities to the maximum, it is possible to keep the material usage at a minimum. In the case of carbon fiber composites, their tensile strength is considerably higher than their compressive strength. This presents an opportunity to explore a design that takes full advantage of tensile forces, leading to the development of a suspended chair.

Traditional standing chairs are designed to transmit compressive forces exerted by a load of a person through the legs. Applying this design approach to carbon fiber composites could result in a dense, material costly product. By allowing the chair to hang instead of stand - a new design expression that properly conveys the material characteristics presents itself.

To realize this concept a novel fabrication system that ensures an effective transmission of tensile forces through the shape had to be developed. Additionally, a computational tool was created in order to inform design decisions and evaluate possible outcomes.

\section{Material System}

Carbon fiber reinforced composites have been extensively employed in the military, aerospace and automobile industries due to their excellent mechanical properties such as high specific strength and stiffness. In spite of their high cost, composites are widely opted because of their tailored properties (Reddy et al. 2017).

Carbon fiber is a versatile material and can be obtained in different formats such as yarn, braids, multiaxial fabrics, non-crimped fabric or unidirectional fabric. The format used in this project, the yarn, is suited for processes like prepregging, filament winding, pultrusion, weaving and braiding [5].

\begin{tabular}{|c|c|c|c|c|c|}
\hline \multicolumn{2}{|c|}{ Material type } & \multirow{2}{*}{$\begin{array}{c}\begin{array}{c}\text { Density } p \\
\left(\mathrm{~kg}^{\prime} \mathrm{m}^{3}\right)\end{array} \\
1760\end{array}$} & \multirow{2}{*}{$\begin{array}{c}\begin{array}{c}\text { Tensile Strength } \\
\sigma_{u}(\mathrm{GPa})\end{array} \\
3.53\end{array}$} & \multirow{2}{*}{$\begin{array}{c}\begin{array}{c}\text { Elastic Modulus } \\
\text { E (GPa) }\end{array} \\
230 \\
\end{array}$} & \multirow{2}{*}{$\begin{array}{c}\begin{array}{c}\text { Breaking Length } \\
\sigma_{u} /(\rho g)(\mathrm{km})\end{array} \\
205\end{array}$} \\
\hline \multirow{3}{*}{ Carbon fiber } & Standard & & & & \\
\hline & High strength & 1820 & 7.06 & 294 & 396 \\
\hline & High modulus & 1870 & 3.45 & 441 & 188 \\
\hline \multirow{2}{*}{ Steel } & 5355 & 7850 & 0.50 & 210 & 6 \\
\hline & Wire & 7850 & 1.77 & 210 & 23 \\
\hline
\end{tabular}

When combined with a thermoset or a thermoplastic matrix, carbon fibers make up a high-performance composite. In a fibrous composite, the fibers are the principal load-carrying constituents, therefore, the mechanical properties of a fibrous composite are, in general, dominated by the fiber characteristics (llankeeran et al. 2012). The mechanical properties of the composite may vary depending on the producers of the resin and the fibers and how the two are combined. As shown in Table 1, the tensile strength of carbon fibers can reach impressive values, as 7,06 GPa, being much higher than steel wires with $1,77 \mathrm{GPa}$, while their densities are much lower, 1820 $\mathrm{Kg} / \mathrm{m} 3$ compared to $7850 \mathrm{Kg} / \mathrm{m} 3$. (Liu et al. 2015).

This project aims to exploit the tensile strength of carbon fiber composites to its maximum by applying it to a minimal design that reflects the material qualities.

\section{Application in Architecture and Design}

Carbon fiber composites have introduced new possibilities within the fields of architecture and design through their inherent capacity to form complex geometries with a programmed behavior. By controlling the directionality of the fibers the performance of a structure can be tailored to specific structural requirements (Prado et al. 2014).

In their research ICD and ITKE combine principles from biomimetics, material science, robotic fabrication, engineering, and architecture to create complex, lightweight fiber composite construction systems. They have explored both component-based fabrication systems as well as on-site continuous fabrication processes. The Elytra Pavilion, a lightweight
Table 1

Mechanical properties of carbon fibers compared with steel materials (Liu et al. 2015) 
glass, and carbon fiber canopy inspired by the protective shell of beetles (Prado et al. 2017) shows how larger fiber constructions can be realized with components. The ICD/ITKE Research Pavilion 2015, a carbon fiber pavilion inspired by the water spider, explores a different method as it was fabricated in-situ with a sensor-driven robotic arm (Doerstelmann et al. 2015).

The Fibre Facade Prototype, developed by the engineer James Solly (ITKE) together with FIBR $\mathrm{GmbH}$, is a facade system demonstrator of carbon fiber reinforced polymer designed to carry the load of glass facade panels and expected wind loads for a building envelope. The visual appearance of the frames is minimal and emphasizes the high performance of the material [1].

Carbon fiber composite in a form of a yarn or a tow has been applied in a few furniture projects. Bertjan Pot's 'Carbon' chair [2] is an example of how traditional design can be revisited with modern materials. In contrast, Korean designer IL Hoon Roh's projects 'Luno' lounge chairs [3] and 'Rami Bench New York' [4] show how new design expressions follow new materials.

With carbon fiber comes a completely new aesthetics for lightweight structures. Although Pot and Roh explore the use of carbon fiber in their projects, they do not fully explore the minimal layouts made possible with the material as demonstrated in Solly's project.

\section{Fabrication methods for filament materials}

Carbon fibers can be, among other processes, winded, woven and braided (Teijin Carbon Europe $\mathrm{GmbH}$. n.d.). Historically the processing of filament materials was executed by trained human hands, until it was automated during the industrial revolution, with for example the invention of the Jacquard loom.

The technological advancements of the past decades have opened up a new range of possibilities with regards to the fabrication for filament structures (Marshall Prado 2016). Automated tailored fiber placement allowed for placing the fiber in the ideal stress-load direction, and has resulted in successful, lightweight design projects, such as the three-legged stool developed at the Leibniz-Institut für Polymerforschung Dresden (Richter et al. 2015). Coreless Filament winding with 6-axis industrial robotic arms (Doerstelmann et al. 2015), aerial robots (Felbrich et al. 2017) and mobile robots (Yablonina et al. 2017) have proven successful winding methods for producing highly customized filament architecture without the use of molds.

In this project, the fibers have to be threedimensionally woven, which requires a complex exchange of material, still not available as a robotic setup. This project serves as a small-scale proof-ofconcept of a fabrication setup that should later be automated through a large-scale robotic setup. Until the computational tool and all fabrication parameters are adjusted, the fabrication is manually operated.

\section{METHODS \\ Design method}

Antoni Gaudí spent his professional career exploring the use of hanging models to obtain shapes in equilibrium. To conduct his experiments Gaudí needed a design tool that allowed him to carry out quick calculations and alter the design at will, obtaining equilibrated forms directly (Huerta 2006). The use of hanging models enabled him to shift from calculating two-dimensional arches with graphic statics to design and calculate spatial three-dimensional vaults. These space-hanging models applied the slicing method firstly used by Hooke in the last quarter of the 17th century, in which the vault is understood as the sum of a series of arches obtained by "slicing" the structure with a certain number of planes (Huerta 2006).

Gaudi's models were constructed hanging, achieving an equilibrated distribution of tensile forces, and when transformed into drawings for construction were flipped, producing a form ideal for compressive forces. As the goal of this project is to achieve the equilibrium shapes for tensile forces, the 
models remain in the initial hanging position, without being flipped afterward.

The proposed design method uses a similar approach to the above-mentioned "slicing" technique. In this case, parallel catenaries are formed by hanging strands with different lengths. While Gaudi shaped his catenaries by attaching sandbags to specific positions, this project uses non-elastic threads to pull each strand into the desired shape. At each carbon strand/thread intersection, a node is created at specific positions in space (Figure 1).

The nodes are then used to weave the catenaries together, forming a surface, which allows for the direct shaping of a $3 \mathrm{~d}$-grid in space.

After being cured, these first fibers are used as formwork, just like the weaving process of a cobweb, where the spider uses the first threads as the supporting structure for further weaving (Ali Askarinejad 2015). With this approach, it is possible to create a complex three-dimensionally weaved surface independent of its frame. In most winding systems, every node is related to a mechanical anchor point (usually a metallic spacer or a bolt). However, in this case with a small number of anchor points in the frame and on the floor, a large number of independent nodes are created

From this design logic, many resulting gridconfigurations can be formed. To enhance the design process and inform the decision making, a computational tool was developed.

\section{Integrated design and form-finding tool}

Initially, a simple computational tool was developed to enable a fast generation of designs. It was scripted in Grasshopper with a custom Python component. The input parameters were the number of fiber intersection points in $\mathrm{y}$ and $\mathrm{x}$-direction connected by lines. The $z$-height of each point could be interactively changed to form the desired chair.

Although this method proved to be useful to some extent, the strategy evolved from the manual adjustment of points to a form finding method that to a larger degree reflected the design and fabrica-

tion methods.

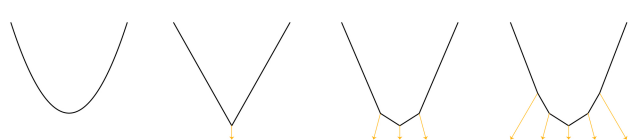

Using the Finite Element Analysis (FEA) plugin Karamba, gravity-based forms following the hanging chain principle were generated. The catenaries were acquired from the form found mesh under gravity (Figure 2). The design of the chair could interactively change by adjusting the mesh resolution and the position of the mesh anchor points. While the $Z$ and $Y$ Axis affected the lateral section (Figure $3 a$ ), the $X$-Axis affected the width of both the seat and backrest (Figure $3 b)$.

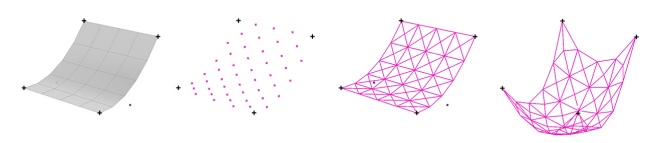

The mechanical properties of a non-cured carbon fiber composite were considered as input. In this case, Young's Modulus ( $25000 \mathrm{kN} / \mathrm{cm} 2$ ) derived from the pure fiber without considering the properties of the resin.
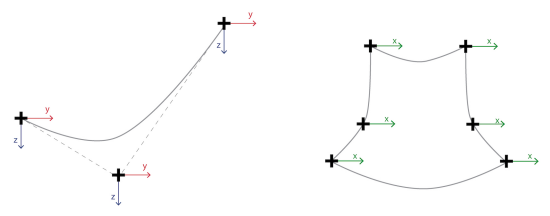

Although computational methods for hanging chain models are well established in the computer graphics community and the animation industry (Kilian 2004), they were rarely targeted for architectural purposes. Axel Kilian applies this method as an interactive tool for designers, enabling a playful exploration of evolving structures (Kilian 2004).
Figure 1

Design principle, each carbon fiber strand is shaped by being pulled in a specific direction

Figure 2

Form finding tool overview: mesh is created with 6 control points. The squared grid is subdivided forming a triangulated mesh. Gravity load is applied to the mesh.

Figure 3

The $Z$ and $Y$-axis affect the lateral section, and the $X$-axis affects the width of the seat and backrest 
Figure 4

Fabrication setup

Figure 5

Fabrication

procedure

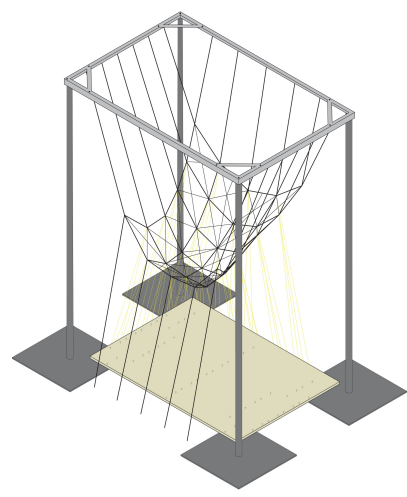

Different from Killian's approach, where he applies different loads to specific elements achieving more complex geometries, the design and fabrication setups proposed here imply in a simple combination of parallel and perpendicular catenaries, what can be obtained digitally considering only the self-weight, without the addition of any extra load. This strategy offered an accessible and fast way to visualize possible outcomes.

\section{Fabrication method}

Parallel strands of carbon fiber pre-impregnated with epoxy resin were hung on nodes attached to the top of a rectangular frame. The length of each strand was predefined. Subsequently, each of them was shaped with multiple non-elastic threads attached to the ground (Figure 4). By differentiating the length and spacing of each tensioning thread the form of the carbon strands were determined (Figure 5). At each fiber-thread intersection, a new node was created, and these were then used to weave the network of fibers between the initial strands composing the seat and backrest.

After curing, all threads and carbon fiber strands that were attached to the ground and the frame were cut away. Finally, thin metal wires were connected to each of the four corners of the chair and the ceiling and allowed it to hang and swing.
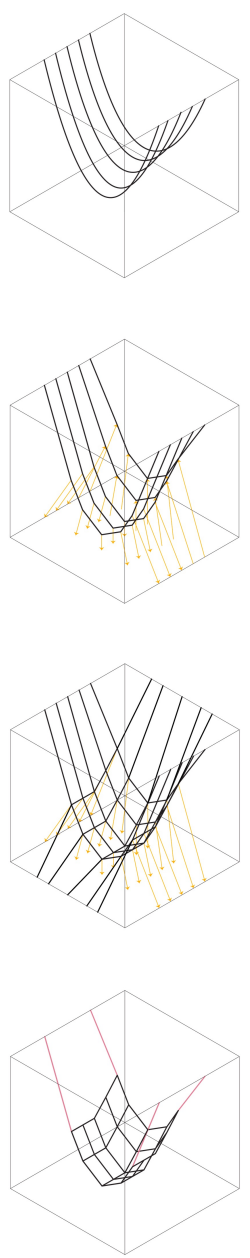


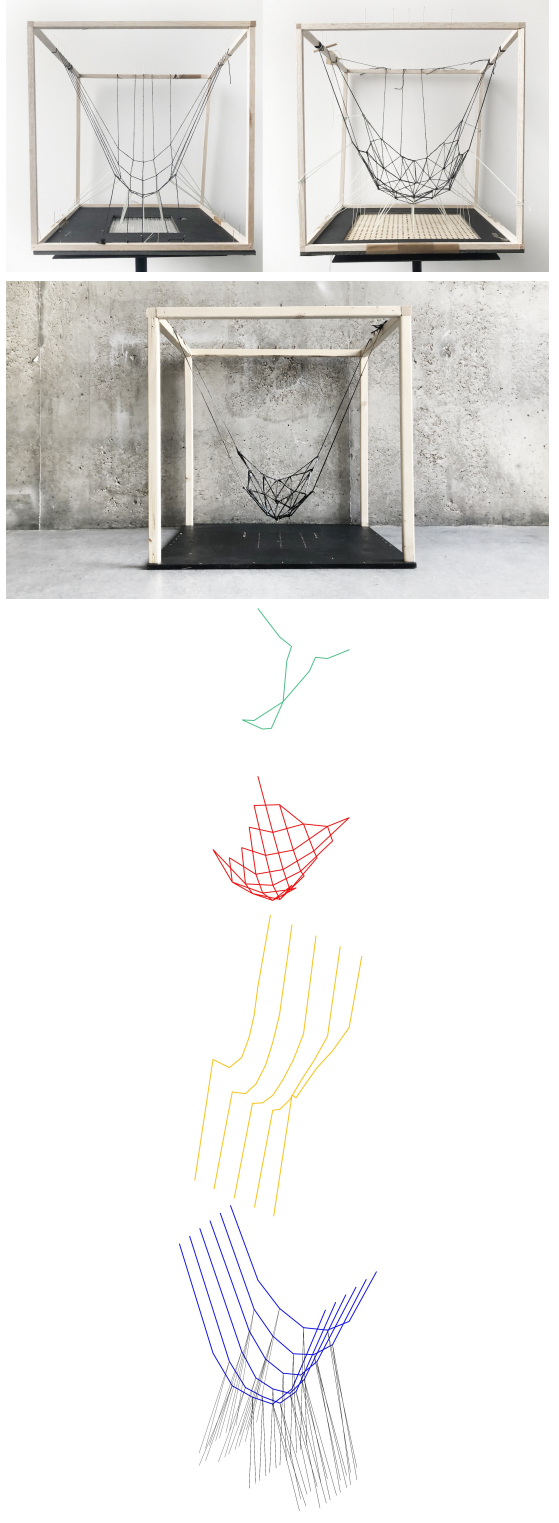

\section{RESULTS}

\section{Case study: The hanging chair} Physical prototypes and material tests

Both the design method and the fabrication process were explored and tested with physical prototypes of different scales and materials. With the making of the prototypes, it was possible to understand the results of different syntax strategies as well as fiber interactions that resulted from it.

Two 1:10 physical models (Figure 6) were developed using a wooden frame, foam board and nonelastic cotton thread impregnated with resin. The purpose of these models was foremost to explore the design outcome, not considering the material properties.

Subsequently a 1:5 model (Figure 7) utilizing the actual material was produced. Important takeaways from this process were that the sliding of the ropes would cause fibers breakage, that the fabrication process must be undertaken with multiple curing sessions, and that it must be confirmed that the spool can pass in between all fiber intersections. Additionally, a better understanding of the winding syntax was acquired.

\section{Full-scale prototype}

In preparation for the full-scale physical prototype, a digital design was generated. It was primarily based on the small scale prototypes and created with the initial version of the form-finding tool. A 4x5 triangular mesh was defined and gravity load was applied, generating its final shape. The obtained design functioned as a guide for the winding sequence and provided the necessary practical information such as the length of the initial carbon strands.

When the digital design was ready and other offsite practical preparations for the fabrication were concluded, a workspace consisting of a frame and a screw hook board was constructed. The frame was composed of four poles connected at the top with a lightweight, rectangular metal frame. In between the poles, a piece of plywood with distributed metal screw hooks was placed. The screw hook board al-
Figure 6

Prototypes 1 \& 2 ,

$1: 10$

Figure 7

Prototype 1:5

Figure 8

Exploded isometry of the four winding steps: initial strands, perpendicular strands, diagonals, reinforcement. 
Figure 9

The prepregg spool, ca $50 \mathrm{~m}$ of carbon fiber

Figure 10

The metallic loops were installed during the fabrication process

Figure 11

The final curing of the chair in room temperature lowed the tensioning threads to be attached to the floor.

To guarantee a clean fabrication process and minimize resin dripping, the carbon fiber yarns (Tenax ${ }^{\circledR}$ E STS40 F13 48K 3200tex) were preimpregnated with epoxy resin ( 3 parts of EPIKOTE Resin MGS LR 135 + 1 part of EPIKURE Curing Agent MGS LH 137, both produced by Hexion Inc. Pot Life: 8 hrs.) wound on spools and frozen (Figure 9). The freezing period varied from short (30min) to long (12hrs), both presenting good results. However, it should be noted that the freezing time of pre-impregnated carbon fiber varies according to the resin type and producer. The freezing stops the hardening process and allows a clean preparation of the composite prior to usage.

When the pre-impregnated carbon fiber spools were ready, the first six strands were wound, shaped and then left to cure for 24 hours in room temperature. Afterward, the perpendicular strands and the diagonals forming the triangulated pattern of the seat were woven over-and-under the cured fibers and left to cure for 24 hours (Figure 11).

Following the second curing, all of the connecting fibers and ropes were cut away. The metallic loops (Figure 10) were installed during the winding process in the four corners of the chair allowing the connection of wires that enabled the chair to hang from the ceiling and swing when installed.

In total, the chair was suspended in air anchored to 17 points from the upper part of the frame and put in tension with 30 screw hooks on the ground. It measured $90 \mathrm{~cm}$ by $90 \mathrm{~cm}$, had an impressive total weight of $300 \mathrm{~g}$ and could hold the weight of a human body (60kg) (Figure 14).

\section{Computational tool 2.0}

After the completion of the physical prototype, it became evident that the design method/process would benefit from a more sophisticated computational application. The initial tool provided an effective way of generating geometries, but not a way of structurally evaluating and optimizing them.
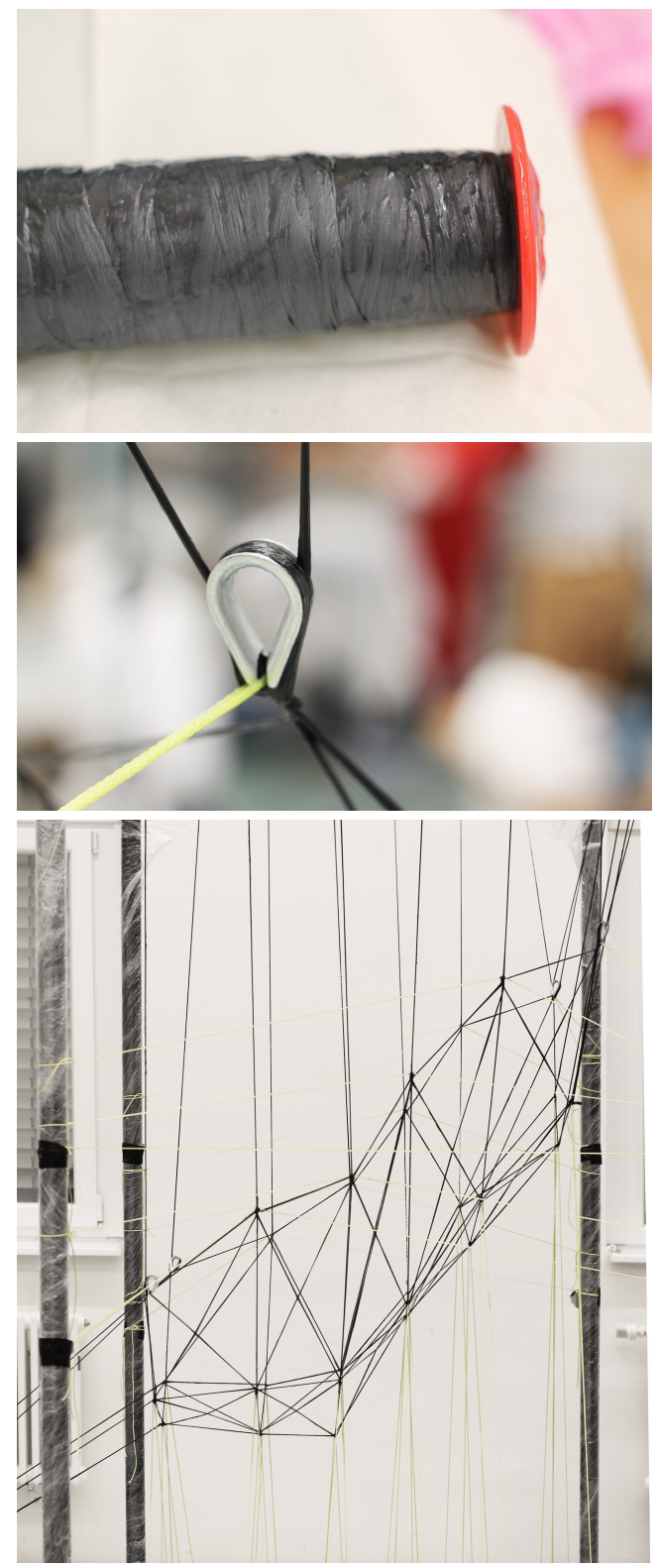

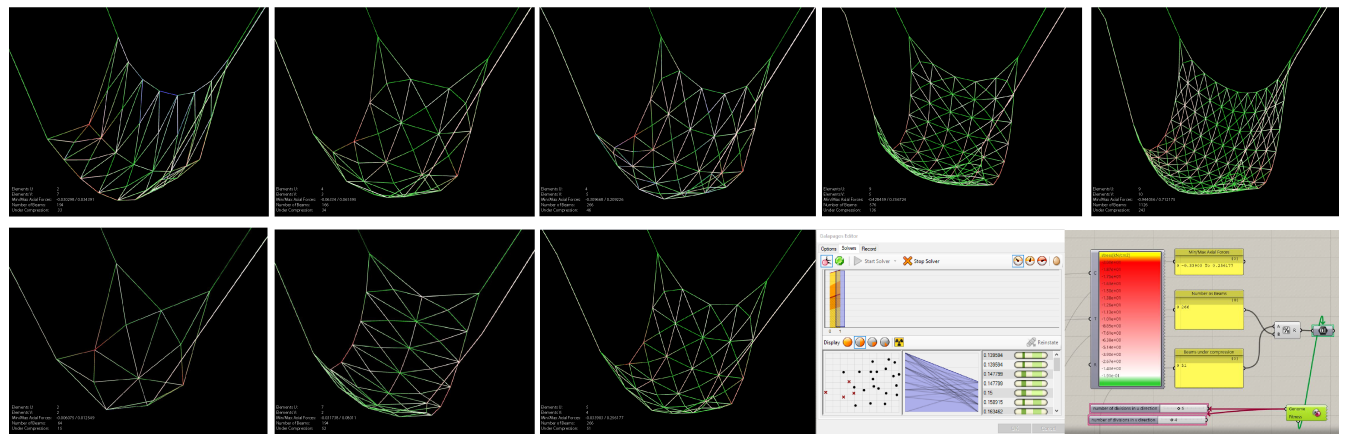

The initial tool was then expanded and resulted in an interactive 3D-modeling interface that combined design intentions with structural optimization.

The structural optimization takes place by disassembling the form-found model in new elements and simulating the deformation of the chair under a load of a human.

From this moment on, the material properties of the model change to the mechanical properties of a cured carbon fiber composite, with Young's Modulus $=8700 \mathrm{kN} / \mathrm{cm} 2$. To enable the chair to realistically deform, the anchor points are transferred to points representing the connection to the ceiling and attached to the previous anchor points with four new elements.

The simulation returns which elements are under tension and compression when deformed. Figure 13 shows an example of a chair model before and after deformation. The results obtained from the FEA analysis were then connected to an evolutionary plugin (Galapagos). Taking into consideration the goal to have the model working under tension, the fitness criteria was set to minimize the percentage of elements under compression. The parameters, or genes, allowed to change the subdivision of the grid, changing the mesh configuration (Figure 12).

The use of a generative algorithm offered a quick validation of different design iterations while guaranteeing structural performance.

\section{DISCUSSION AND CONCLUSION}

The built chair was incredibly light (300 grams) and able to carry the weight of a person.

Nonetheless, the syntax and topology optimization still need further development and some failure points were noticed.

With the proposed fabrication setup it was not possible to accurately convert the digital design to the physical chair. It proved a challenge to symmetrically pull the tensioning threads without causing individual fibers to break.

Although the digital model provided the target length of all fiber strands and tensioning threads,

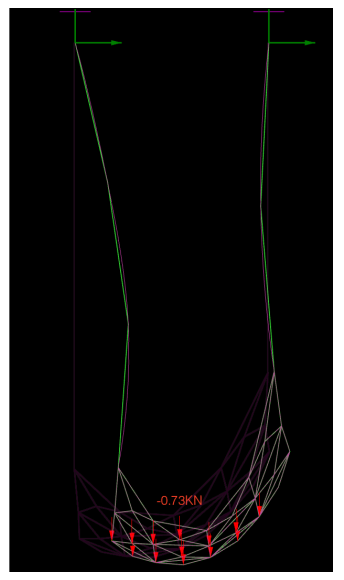

Figure 13

Comparison of the model before and after structural simulation 
Figure 14

The final prototype
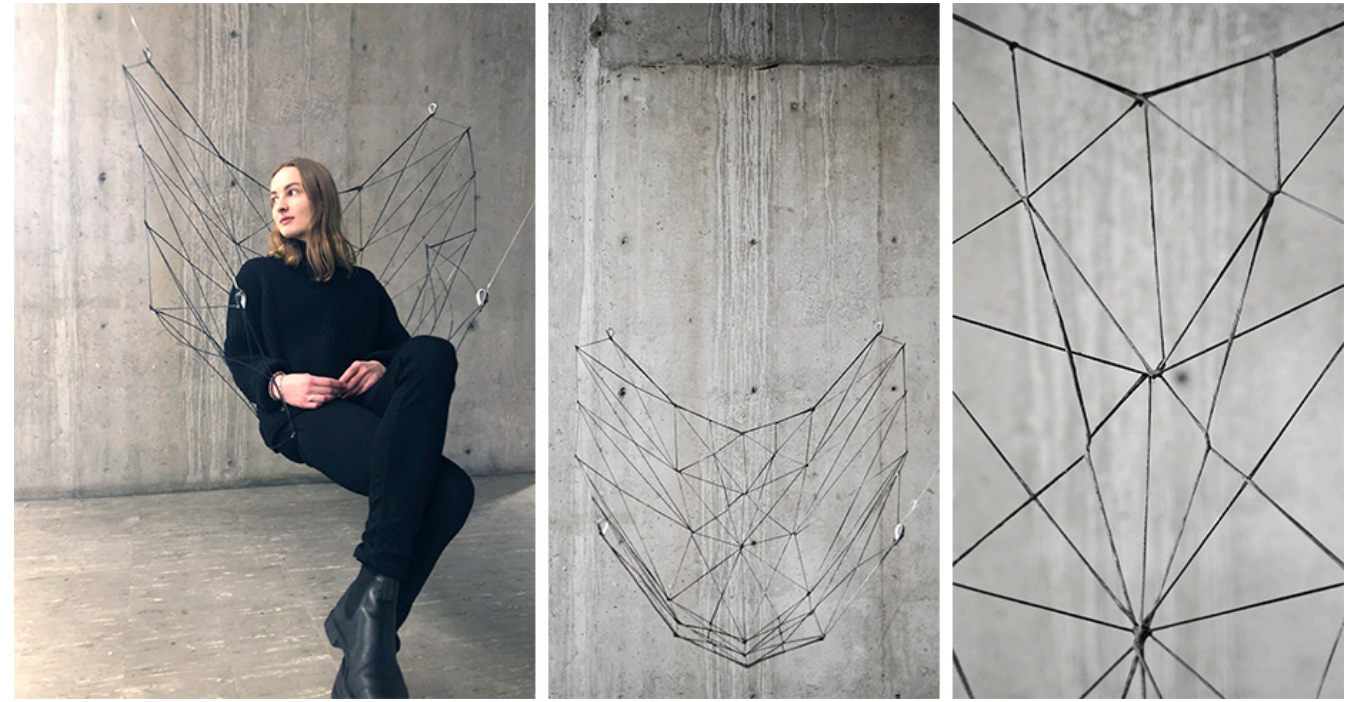

it did not return the angle between them and the hooks. Consequently, the placement of some threads had to be improvised during fabrication, which lead to imprecision. This data could be implemented in the further development of the tool.

Several of these challenges could have been tackled with a complex robotic fabrication setup consisting of two robots collaborating. However, as mentioned, the setup does not exist and it was not the goal of this project to develop one. Nonetheless, it could be an interesting continuation of the project to develop such a setup.

Aiming for a more informed structural optimization method, the future development of the tool could consider the use of a multi-objective evolutionary plugin. It would allow the analysis of the elements under compression together with the values of maximum displacement and produce a more precise result.

Another relevant next step would be to research the use of more environmentally friendly filament materials such as natural fibers or carbon fibers retrieved from lignin. Because the source of these ma- terials are renewable, they could represent a more sustainable solution for lightweight filament structures (Dahy, 2019).

Gaudí innovates by using the concept of catenary arches to integrate the structural design in the process of design. This project aims to make the realization of equilibrated forms accessible, and to combine design intentions and structural optimization through physical and digital models.

The technological advancements of the past decades have allowed us to integrate the different aspects of a design process. By alternating between digital and physical design spaces, the design process can be significantly more informed. The goal of this paper is to present a solution in which the material is not a mere bystander, but plays the main role by informing every aspect of the design and fabrication process, and with this hopes to contribute to the discussion on integrative computational design.

\section{AUTHORSHIP INFORMATION}

Duque Estrada and Wyller contributed equally to this paper. Concept, design, methods and prototyping of 
the project, as well as writing the paper was done by the two together. Hanaa Dahy edited the paper and supervised the educational course in which this prototype was produced.

\section{ACKNOWLEDGMENTS}

The project was developed in the seminar Material and Structure offered by The Department of Biobased Materials and Materials Cycles in Architecture (BioMat) at the University of Stuttgart.

We would like to thank our tutor Piotr Baszyński for support during the development of the project.

We would also like to show our gratitude to Serban Bodea, Niccolo Dambrosio, Marta Gil Peréz and Christoph Seichmeister at the Institute of Computational Design (ICD) for providing us with materials and space for prototyping, as well as Bas Rongen at the Institute of Building Structures and Structural Design (ITKE) for help with Karamba3d.

\section{REFERENCES}

Askarinejad, A. 2015 'Spatial Nets: the Computational and Material Study of Reticular Geometries', ACADIA 2015, pp. 123-135

Dahy, H. 2019, 'Natural Fibre-Reinforced Polymer Composites (NFRP) Fabricated from Lignocellulosic Fibres for Future Sustainable Architectural Applications, Case Studies: Segmented-Shell Construction, Acoustic Panels, and Furniture', Sensors, 19(3), p. 738

Dörstelmann, M., Koslowski, V., Prado, M., Schieber, G. Vasey, L., Knippers, J. and Menges, A. 2015, 'ICD/ITKE Research Pavilion 2014-15: Fibre Placement on a Pneumatic Body Based on a Water Spider Web', Architectural Design, 85(5), pp. 60-65

Dörstelmann, M. and Prado, M. 2016, 'Material Performance: Fibrous Tectonics \& Architectural Morphology', in Menges, A. (eds) 2016, Integrative Design and Fabrication Methodologies for Fibrous Systems, Harvard Graduate School of Design, pp. 18-24

Dörstelmann, M., Prado, M., Solly, J., Menges, A. and Knippers, J. 2017, 'ELYTRA FILAMENT PAVILION', Fabricate 2017, pp. 224-231

Felbrich, B., Frueh, N., Prado, M., Saffarian, S., Solly, J., Vasey, L., Knippers, J. and Menges, A. 2017 'MultiMachine Fabrication: An Integrative Design Process Utilising an Autonomous UAV and Industrial Robots for the Fabrication of Long Span Composite Structures', ACADIA, pp. 248-259

Huerta, S. 2006, 'Structural Design in the Work of Gaudi', Architectural Science Review, 49(4), pp. 324-339

Ilankeeran, P.K., Mohite, P.M. and Kamle, S. 2012, 'Axial Tensile Testing of Single Fibres', Modern Mechanical Engineering, 02(04), pp. 151-156

Kilian, A. 2004 'Linking Hanging Chain Models to Fabrication', AIA/ACADIA, pp. 110-125

Liu, Y., Zwingmann, B. and Schlaich, MS 2015, 'Carbon Fiber Reinforced Polymer for Cable Structures - A Review', Polymers, 7(10), pp. 2078-2099

Prado, M., Dörstelmann, M., Schwinn, T., Menges, A. and Knippers, J. 2014, 'Core-less Filament Winding', Robotic Fabrication in Architecture, Art and Design 2014, pp. 275-289

Reddy, C.V., Babu, P.R., Ramnarayanan, R. and Das, D. 2017, 'Mechanical Characterization Of Unidirectional Carbon And Glass/Epoxy Reinforced Composites For High Strength Applications', Materials Today: Proceedings, 4(2), pp. 3166-3172

Richter, E., Spickenheuer, A., Bittrich, L., Uhlig, K. and Heinrich, G. 2015, 'Applications of Variable-Axial Fibre Designs in Lightweight Fibre Reinforced Polymers', Materials Science Forum, 825-826, pp. 757-762

Yablonina, M., Prado, M., Baharlou, E., Schwinn, T. and Menges, A. 2017, 'MOBILE ROBOTIC FABRICATION SYSTEM FOR FILAMENT STRUCTURES', Fabricate 2017, pp. 202-209

[1] https://www.itke.uni-stuttgart.de/archives/9873

[2] http://www.bertjanpot.nl/work/carbon-chair/

[3] http://ilhoon.com/luno/

[4] http://ilhoon.com/rami-bench-ny/

[5] https://www.teijincarbon.com/fileadmin/PDF/Date nbl\%C3\%A4tter_en/Filament-Product_programm_EU _v27_2018-06-27_EN.pdf. 